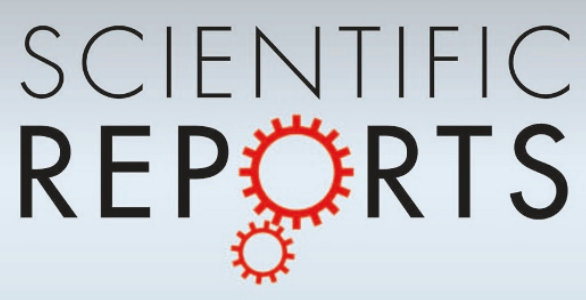

OPEN

\section{SUBJECT AREAS:}

RISK FACTORS

PROTEIN-PROTEIN INTERACTION

NETWORKS

Received

17 September 2014

Accepted

23 February 2015

Published

16 March 2015

Correspondence and requests for materials should be addressed to

M.-G.P. (mgpang@ cau.ac.kr)

\title{
Bisphenol-A Affects Male Fertility via Fertility-related Proteins in Spermatozoa
}

\author{
Md Saidur Rahman, Woo-Sung Kwon, June-Sub Lee, Sung-Jae Yoon, Buom-Yong Ryu \& Myung-Geol Pang
}

Department of Animal Science and Technology, Chung-Ang University, Anseong, Gyeonggi-do 456-756, Republic of Korea.

The xenoestrogen bisphenol-A (BPA) is a widespread environmental contaminant that has been studied for its impact on male fertility in several species of animals and humans. Growing evidence suggests that xenoestrogens can bind to receptors on spermatozoa and thus alter sperm function. The objective of the study was to investigate the effects of varying concentrations of BPA $(0.0001,0.01,1$, and $100 \mu \mathrm{M}$ for $6 \mathrm{~h})$ on sperm function, fertilization, embryonic development, and on selected fertility-related proteins in spermatozoa. Our results showed that high concentrations of BPA inhibited sperm motility and motion kinematics by significantly decreasing ATP levels in spermatozoa. High BPA concentrations also increased the phosphorylation of tyrosine residues on sperm proteins involved in protein kinase A-dependent regulation and induced a precocious acrosome reaction, which resulted in poor fertilization and compromised embryonic development. In addition, BPA induced the down-regulation of $\beta$-actin and up-regulated peroxiredoxin-5, glutathione peroxidase 4, glyceraldehyde-3-phosphate dehydrogenase, and succinate dehydrogenase. Our results suggest that high concentrations of BPA alter sperm function, fertilization, and embryonic development via regulation and/or phosphorylation of fertility-related proteins in spermatozoa. We conclude that BPA-induced changes in fertility-related protein levels in spermatozoa may be provided a potential cue of BPA-mediated disease conditions.

isphenol-A (BPA) is an endocrine disruptor (ED) that possesses weak estrogenic, anti-androgenic, and anti-thyroid activities, and it is a prominent environmental pollutant due to its use in the manufacture of food packaging, industrial materials, and dental sealant ${ }^{1}$. Owing to its extensive use, toxicity, and environmental persistence, BPA has become a significant public health concern. The major route of BPA exposure for humans and animals is ingestion of contaminated food and water, but exposure can also occur via dermal and respiratory routes ${ }^{2}$.

BPA is associated with the impairment of male reproductive function, as well as diabetes, obesity, cardiovascular disease, thyroid dysfunction, developmental disorders, miscarriages, female reproductive abnormality, and cancer ${ }^{3}$. Most studies on BPA in the context of male reproduction/fertility have been conducted in vivo, and these studies have consistently reported its developmental toxicity to spermatozoa via DNA damage ${ }^{4}$, DNA methylation $^{5}$, oxidative stress ${ }^{6}$, and lipid peroxidation ${ }^{7}$, among other mechanisms. While few in vivo studies have shown an association between BPA and spermatozoa functional parameters, BPA has been linked to changes in motility ${ }^{8}$, morphology ${ }^{9}$, and intracellular ATP content ${ }^{6}$.

Earlier studies have described the presence of nongenomic estrogen receptors on sperm cell membranes, which interfere with sperm functions upon stimulation with estradiol ${ }^{10-12}$. These results show that spermatozoa may represent a valid model for studying the effects of estrogenic EDs like BPA in vitro. This model may allow further exploration of previous in vivo findings via investigation of the effects of BPA on the functional maturation process of mammalian spermatozoa, which includes motility, hyperactivation, capacitation, and the acrosome reaction. This functional maturation process is important because it confers the ability to fertilize oocytes ${ }^{13}$. The effect of BPA on consequent fertilization and embryo development should also be investigated.

The recent application of proteomic approaches has led to the discovery of several spermatozoan proteins, some of which have been associated with fertility ${ }^{14,15}$. However, the precise functions of spermatozoan proteins in fertility/infertility are not well understood ${ }^{16}$. It is difficult to draw conclusions from the results of studies conducted in models that do not use spermatozoa, because proteins can perform functions in the whole organism that differ from their functions in spermatozoa ${ }^{17}$. Thus, to understand the molecular mechanisms that regulate sperm function under environmental stress, intensive studies on individual protein function are needed. Therefore, the objectives of the present study were as follows: (1) to determine whether BPA exposure alters spermatozoa function and identify the underlying molecular mechanisms and (2) to investigate the function of fertility-related proteins in spermatozoa under BPA exposure in vitro. We selected the following fertility-related proteins based 

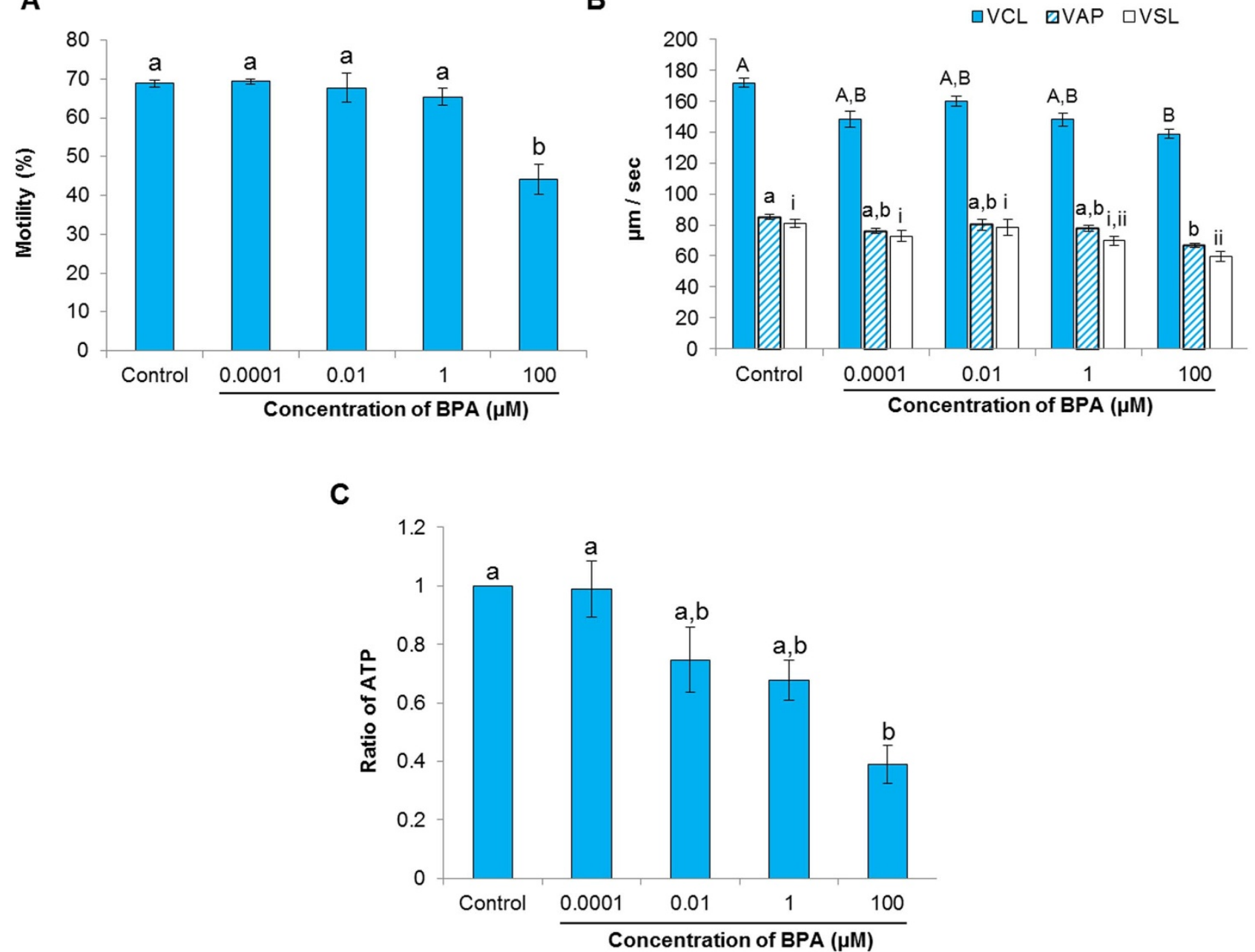

Figure $1 \mid$ Effect of bisphenol-A (BPA) on motility parameters and intracellular ATP levels of spermatozoa. (A) Differences in spermatozoa motility after incubation with different concentrations of BPA compare to control. (B) Differences in motion kinematics parameters such as curvilinear velocity (VCL, blue bar), average path velocity (VAP, stripped bar), and straight line velocity (VSL, open bar) between the control and BPA-treated samples. (C) Differences in ATP levels between the control and BPA-treated samples. Data are presented as mean \pm SEM (4 replicates). Values with different superscript characters $\left({ }^{\mathrm{A}, \mathrm{B}, \mathrm{a}, \mathrm{b}, \mathrm{i}, \mathrm{ii}}\right)$ indicate significant difference between the control and treatment groups as determined by one-way ANOVA $(P<0.05)$.

on previous results: $\beta$-actin ${ }^{18}$, peroxiredoxin-5 (PRDX5) $)^{19}$, glutathione peroxidase $4(\mathrm{GPX} 4)^{20}$, glyceraldehyde-3-phosphate dehydrogenase $(\mathrm{GAPDH})^{21}$, and succinate dehydrogenase $(\mathrm{SDHB})^{22}$. Additionally, we evaluated the functional interactions of the selected fertility-related proteins in spermatozoa with regard to the regulation of sperm function, fertilization, and the fate of the embryo.

\section{Results}

Effect of BPA on sperm motility and motion kinematics. Sperm motility parameters are associated with fertilization and pregnancy rates $^{23-25}$; therefore, the effect of BPA on sperm motility parameters was evaluated. An initial time-dependent experiment (ranging from 0.5 to $8 \mathrm{~h}$ ) was conducted to detect sperm motility in the control group and the high-concentration $(100 \mu \mathrm{M})$ treatment group to allow us to choose the optimal incubation time (see Supplemental Material, Fig. S1). Motility parameters were measured following $6 \mathrm{~h}$ of incubation with ascending concentrations of BPA $(0.0001,0.01,1$, and $100 \mu \mathrm{M})$. The percentage of motile spermatozoa decreased significantly in the presence of $100 \mu \mathrm{M}$ BPA $(P<0.05$; Fig. 1A). $\mathrm{BPA}$ at $100 \mu \mathrm{M}$ also significantly reduced curvilinear velocity, average path velocity, and straight-line velocity $(P<0.05$; Fig. 1B).

The effect of BPA on intracellular ROS content and ATP levels in spermatozoa. To evaluate the effect of BPA on intracellular ROS content and ATP levels in spermatozoa, both were quantitatively detected as described in the Materials and Methods. A significant decrease in ATP production occurred in spermatozoa treated with $100 \mu \mathrm{M} \mathrm{BPA}(P<0.05$; Fig. $1 \mathrm{C})$. However, a non-significant change in ROS production was observed in spermatozoa treated with BPA in comparison with the control (Supplemental Material, Fig. S2).

Effect of BPA on sperm capacitation status. The capacitation status of mammalian spermatozoa is a useful predictor of male fertility ${ }^{26-28}$. In the present study, a dual-staining method was performed to evaluate changes in CTC fluorescence patterns related to sperm capacitation due to in vitro exposure to BPA. Increased numbers of acrosome-reacted (AR pattern) spermatozoa were noted after treatment with $100 \mu \mathrm{M}$ BPA $(P<0.05$; Fig. $2 \mathrm{~B})$. In contrast, the number of capacitated (B pattern) spermatozoa showed a nonsignificant tendency to decrease as the BPA concentration increased (Fig. 2C). BPA treatment also dose-dependently increased the number of non-capacitated (F pattern) spermatozoa, with marked effects achieved using $100 \mu \mathrm{M}$ BPA $(P<0.05$; Fig. 2D).

BPA-induced protein kinase A (PKA)-dependent phosphorylation of tyrosine residues on proteins in spermatozoa. To evaluate the molecular mechanism underlying the BPA-induced acrosome reaction, we measured PKA activity and phosphotyrosine level in 
A
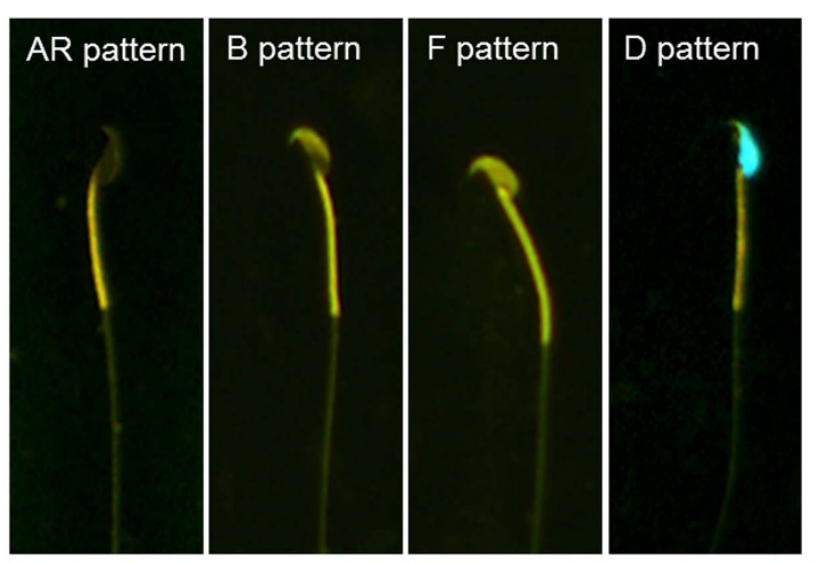

C

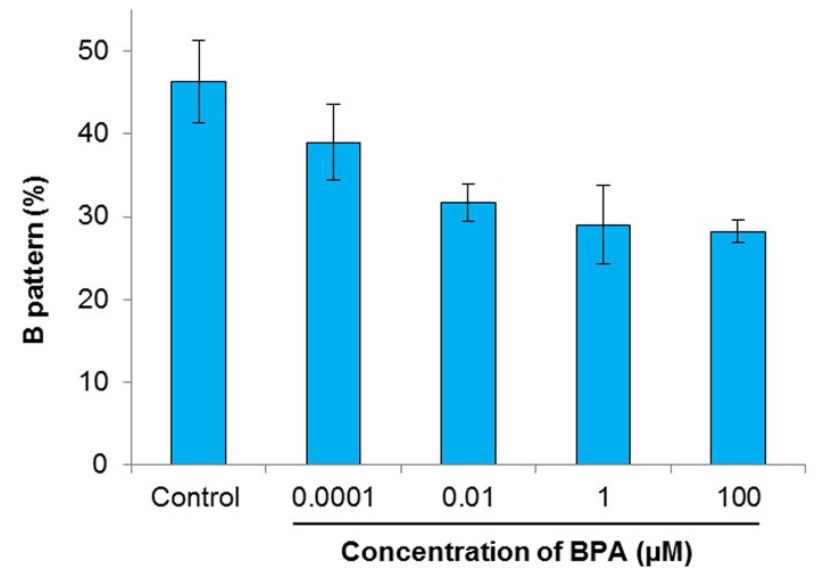

B

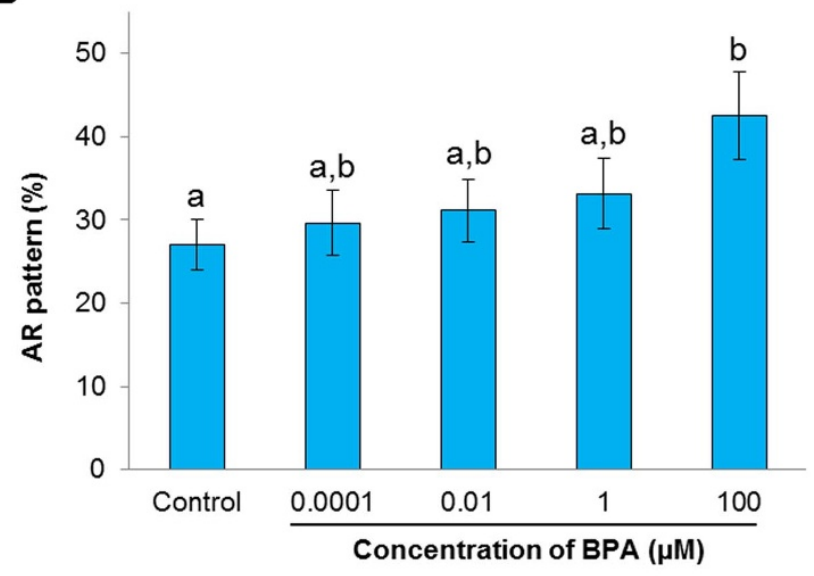

D

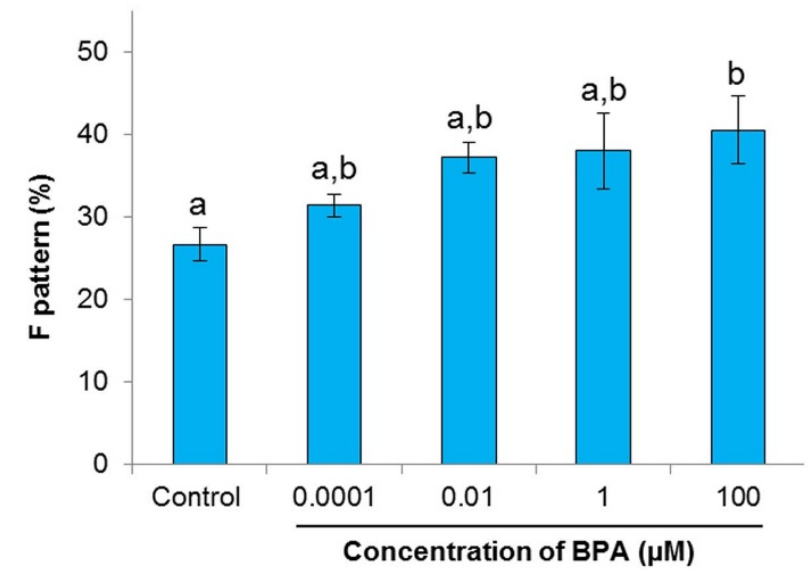

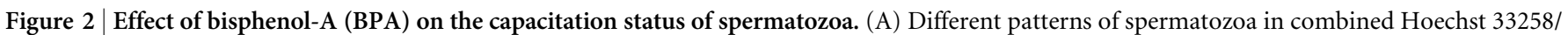

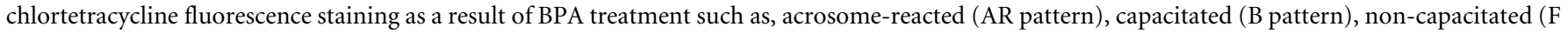

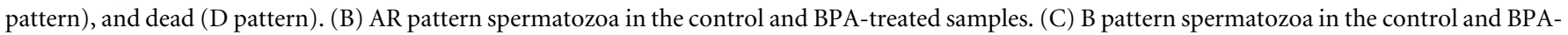

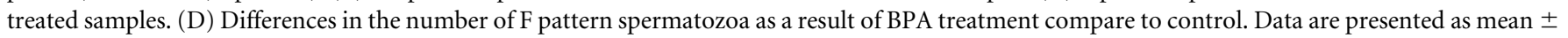
SEM (3 replicates). Values with different superscript characters $\left({ }^{\mathrm{a}, \mathrm{b}}\right)$ indicate significant difference between the control and treatment groups as determined by one-way ANOVA $(P<0.05)$.

spermatozoa by western blotting ${ }^{25}$. Our results showed that the levels of 6 different PKA substrate species (approximately 16, 25, 26, 50, 51, and $100 \mathrm{kDa}$ ) increased dose-dependently compared with their levels in the control cells, with particularly noticeable increases in the species weighing approximately $25,26,50$, and $51 \mathrm{kDa}(P<0.05$; Fig. $3 \mathrm{~A}$, B). Similarly, the tyrosine-phosphorylated species of approximately 24 and $100 \mathrm{kDa}$ increased significantly due to BPA treatment compared with the corresponding levels in the control group $(P<0.05$; Fig. 3C, D).

Effect of BPA on fertilization and embryonic development. Next, we used an in vitro fertilization system to evaluate the effect of BPA on fertilization and early embryonic development. The rates of cleavage (fertilization, Fig. 4A) and blastocyst (early embryonic development, Fig. 4A) formation were significantly decreased in spermatozoa treated with $100 \mu \mathrm{M}$ BPA ( $P<0.05$; Fig. 4B).

Effect of BPA on selected fertility-related proteins in spermatozoa. In this study, we chose several important fertility-related proteins (cytoskeletal $\beta$-actin, the glycolytic enzyme GAPDH, the electron transport chain enzyme SDHB, and stress response proteins GPX4 and PRDX5) to examine their functions in spermatozoa by western blotting due to BPA exposure. $\beta$-actin was effectively down-regulated by BPA, whereas the abundance of other proteins increased dosedependently (Fig. 5A, B). Interestingly, significant changes in protein density occurred primarily after treatment with $100 \mu \mathrm{M}$ BPA (Fig. 5A, B).

Functional analysis of fertility-related proteins. An additional objective of the present study was to evaluate the functional interactions of the selected fertility-related proteins in spermatozoa. Detailed analysis of the cellular processes influenced by the selected proteins was performed using Pathway Studio version 9.0 (Fig. 6). The selected fertility-related proteins were putatively involved to areas related to sperm function, including ROS and energy metabolism, motility, hyperactivation, and the acrosome reaction, as well as cell growth, spermatogenesis, male fertility, and pathological conditions.

\section{Discussion}

Estrogenic EDs like BPA are capable of binding with receptors on spermatozoa and thus affect sperm functions ${ }^{11,12}$. We used a comprehensive in vitro testing system to determine whether BPA interferes with sperm function, fertilization, and embryo development. Additionally, to evaluate the manner in which multiple proteins function in spermatozoa under BPA exposure, we monitored the 
A

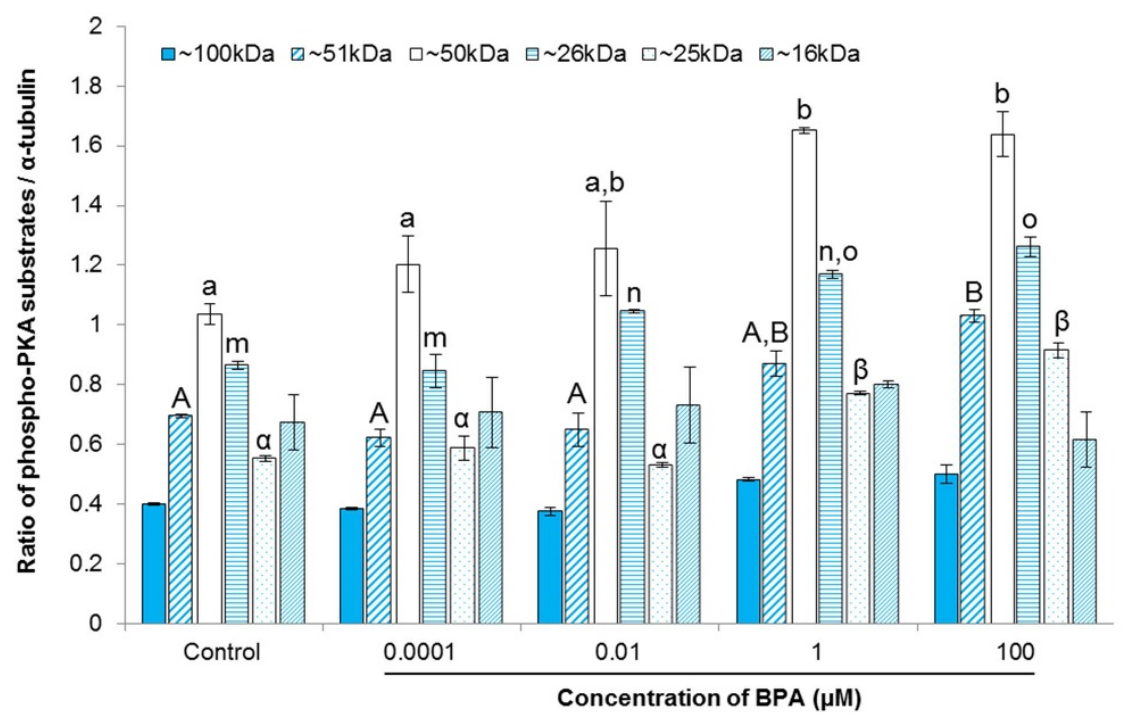

C

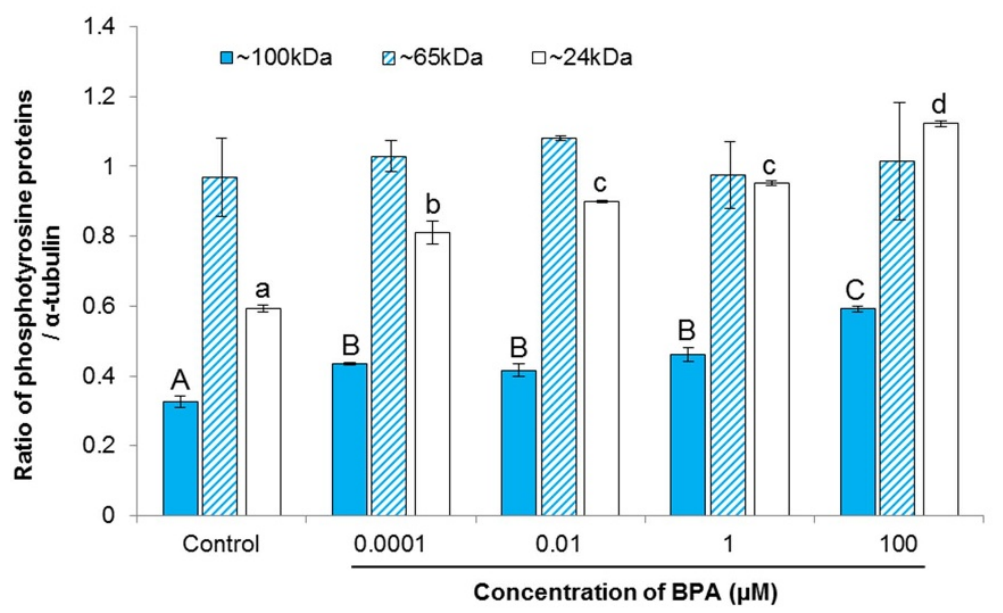

B

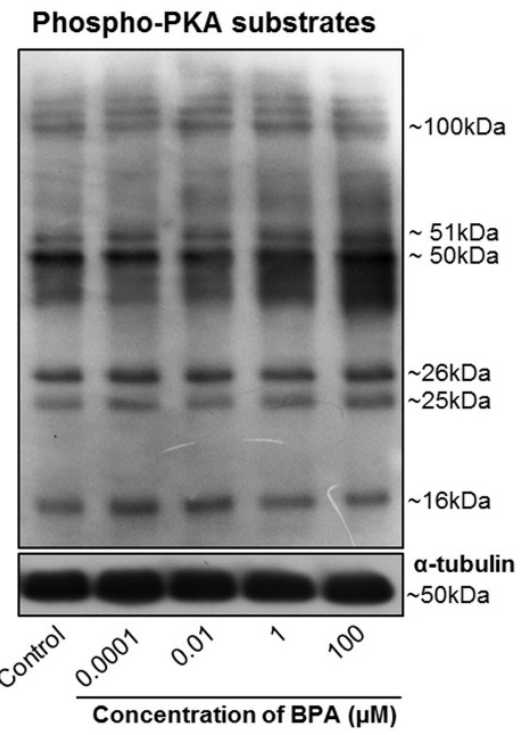

D

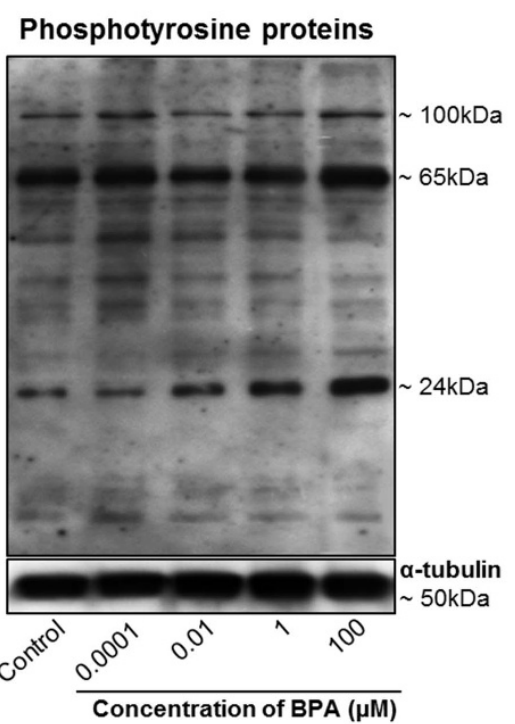

Figure 3 Effect of bisphenol-A (BPA) on protein kinase A (PKA) activity and tyrosine phosphorylation in spermatozoa. (A) Density of phospho-PKA substrates (approximately 100, 51, 50, 26, 25, and $16 \mathrm{kDa}$ ) in control and BPA-treated spermatozoa. (B) Phospho-PKA substrates were probed with an anti-phospho-PKA antibody. Lane 1: control; lane 2: $0.0001 \mu \mathrm{M}$ BPA; lane 3: $0.01 \mu \mathrm{M} \mathrm{BPA}$; lane 4: $1 \mu \mathrm{M}$ BPA; and lane 5: $100 \mu \mathrm{M}$ BPA. (C) Density of phosphotyrosine proteins (approximately 100, 65, and $24 \mathrm{kDa}$ ) in the control and BPA-treated samples. (D) Phosphotyrosine proteins were probed with PY 20 antibody. Lane 1: control; lane 2: $0.0001 \mu \mathrm{M}$ BPA; lane 3: $0.01 \mu \mathrm{M} \mathrm{BPA}$; lane 4: $1 \mu \mathrm{M} \mathrm{BPA}$; and lane 5: $100 \mu \mathrm{M}$ BPA. Data are presented as mean \pm SEM (4 replicates). Values with different superscript characters $(\mathrm{A}, \mathrm{B}, \mathrm{C}, \mathrm{a}, \mathrm{b}, \mathrm{B}, \mathrm{d}, \mathrm{\alpha}, \mathrm{\beta}, \mathrm{m}, \mathrm{n}, \mathrm{o})$ indicate significant difference between the control and treatment groups as determined by one-way ANOVA $(P<0.05)$.

functions of selected fertility-related proteins by western blotting. Furthermore, we explored the functional interactions of these proteins in spermatozoa in the context of male fertility and other health conditions.

Our results demonstrated that $100 \mu \mathrm{M}$ BPA significantly decreased sperm motility and motion kinematic parameters following $6 \mathrm{~h}$ of incubation (Fig. 1A, B). Similar effects of BPA on sperm motility have been shown in rat $^{4}$ and mice ${ }^{9}$ in vivo; however, in vitro exposure data on mammalian species have not been reported. Accordingly, decreased motility after exposure to $100 \mu \mathrm{M}$ BPA was associated with decreased ATP levels in spermatozoa (Fig. 1C). The energy (ATP) used for sperm motility is mostly contributed by mitochondrial respiration ${ }^{21}$; therefore, if mitochondrial respiration does not produce sufficient ATP, sperm motility might decrease.
Capacitation and the acrosome reaction are prerequisites for oocyte fertilization by spermatozoa ${ }^{17,26}$, and both events are linked with modifications in plasma membrane fluidity, intracellular ionic concentrations, cAMP, tyrosine phosphorylation, and PKA activity $^{29}$. Here, we have shown that the addition of BPA to spermatozoa leads to a dose-dependent induction of the acrosome reaction, with a particularly marked effect produced by $100 \mu \mathrm{M}$ BPA (Fig. 2B). It has been reported that exposure of human spermatozoa to $1 \mu \mathrm{M}$ of BPA for $2 \mathrm{~h}$ did not affect the acrosome reaction ${ }^{30}$; this difference with our results was likely due to differences in species, BPA concentrations, and the time of incubation. Additionally, we demonstrated that increased PKA and tyrosine phosphorylation activity accompany the acrosome reaction in spermatozoa (Fig. 3A, C). Indeed, PKA activity directly affects the tyrosine phosphorylation status of sperm proteins during capacitation and the acrosome reaction ${ }^{25,29,31}$. 
A

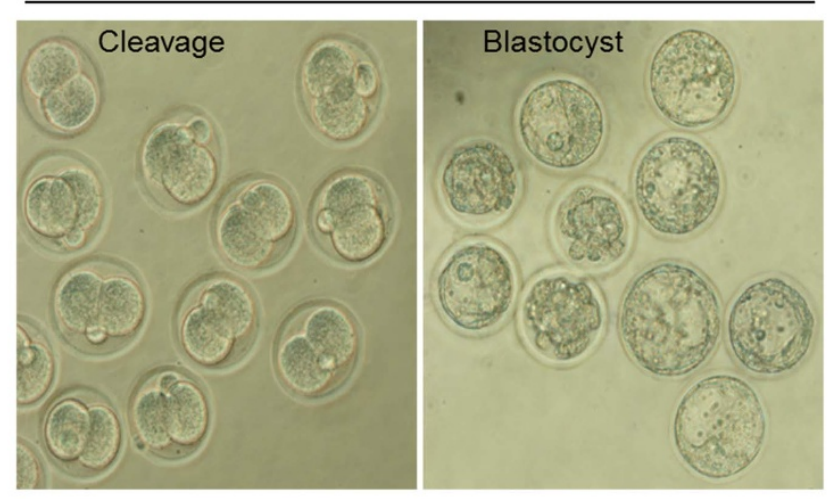

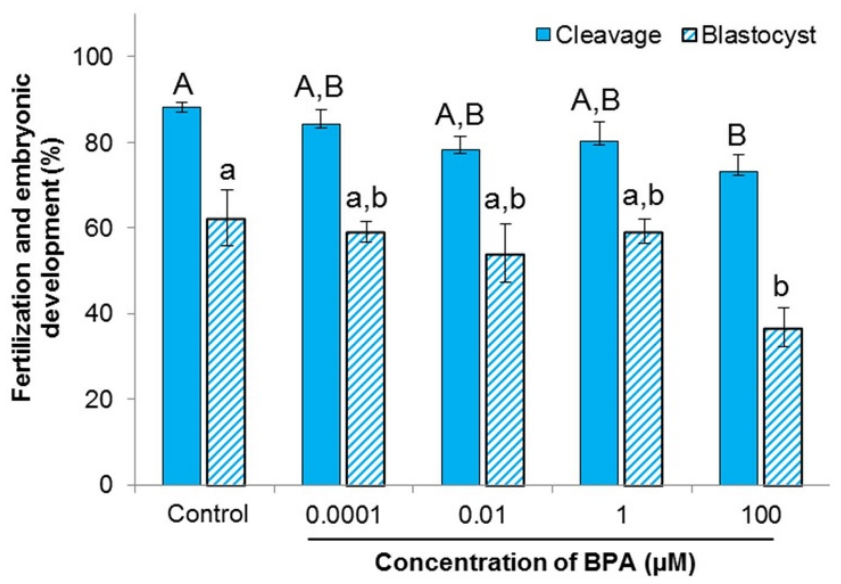

Figure $4 \mid$ Effect of bisphenol-A (BPA) on fertilization and embryonic development. (A) Representative image of cleavage (2 blastomeres containing polar bodies) and blastocyst (embryonic development at day 5). (B) The rate of fertilization (cleavage) and embryonic development (blastocyst) in control and BPA-treated samples (blue bar: cleavage rate; stripped bar: blastocyst rate). Data are presented as mean \pm SEM (3 replicates). Values with different superscript characters $\left({ }^{\mathrm{A}, \mathrm{B}, \mathrm{a}, \mathrm{b}}\right)$ indicate significant difference between the control and treatment groups as determined by one-way ANOVA $(P<0.05)$.

Therefore, taken together with previously published results, our findings suggest that high concentrations of BPA modulate cellular tyrosine phosphorylation by regulating PKA activity, which mediates the acrosome reaction.

Another novel finding of this study is the significant inhibitory effect of $100 \mu \mathrm{M}$ BPA on fertilization and early embryonic development (Fig. 4B), which might be mediated by decreased sperm motility or premature triggering of the acrosome reaction. It has been reported that spermatozoa must exhibit proper movement in fluids to reach and fertilize the egg; thus, motility is a prime factor in successful conception ${ }^{13}$. However, the acrosome reaction is also important for fertilization ${ }^{26}$. Other studies have suggested that a premature acrosome reaction changes mitochondrial function, motility, and chromatin decondensation, and ultimately affect sperm viability and fertility ${ }^{32,33}$. A similar conclusion was made by Oliveira et al. ${ }^{34}$ and Rahman et al. ${ }^{25}$ after exposure of spermatozoa to the lead chloride and sodium nitroprusside, respectively. Collectively, our findings support the notion that a high concentration of approximately $100 \mu \mathrm{M}$ BPA can produce a profound effect on critical sperm functions and fertilization, which has considerable implications for male fertility.

Recently, proteomic approaches have identified several proteins in spermatozoa $^{15,35}$. However, individual protein functions need to be assessed to allow for better understanding of the complex mechanisms involved in sperm function ${ }^{16}$. In this study, we investigated a group of selected fertility-related proteins in spermatozoa under BPA exposure, and found that $\beta$-actin was down-regulated in spermatozoa by high concentrations of BPA, whereas other proteins, including GAPDH, GPX4, PRDX5, and SDHB, were up-regulated (Fig. 5A, B). Protein down-regulation in spermatozoa due to environmental changes in vitro is well established ${ }^{36,37}$; however, the manner in which protein levels are increased due to environmental changes remains to be elucidated.

Mature spermatozoa are thought to have lost their functional ability in transcription and translation, and are therefore incapable of protein synthesis ${ }^{38,39}$. However, some reports suggest that spermatozoa are capable of using mRNA transcripts for protein translation during their functional maturation ${ }^{40,41}$. This discrepancy aside, it is well accepted that spermatozoa acquire their functionality via post-translational protein modifications such as phosphorylation $^{37-42}$. Protein phosphorylation is a common cellular mechanism whereby the addition of a negatively charged phosphate can alter the conformation of a target protein, and thus regulate its function ${ }^{43}$. Although serine, threonine, and tyrosine protein phosphorylation in spermatozoa have been studied, tyrosine phosphorylation has been shown to be a crucial, key activator of spermatozoa signaling pathways $^{44}$. In this study, BPA increased tyrosine phosphorylation (Fig. 3C), which could mediate changes in the abundance of particular phosphorylated proteins. Taken together with a recent study conducted by Wang et al. ${ }^{37}$, our results suggest that exposure to environmental toxicants like BPA induces protein down-regulation and phosphorylation in spermatozoa, ultimately inhibiting their ability to function.

Actin is one of the most abundant cytoskeletal proteins in spermatozoa, and it is localized in the acrosome, equatorial region, and tail $^{18}$. The localization of actin in the tail indicates its function in sperm motility, whereas actin in the head may be involved in the acrosome reaction ${ }^{18}$. Polymerization of globular actin to filamentous actin is critical for capacitation, and this process is regulated by PKA activity and protein tyrosine phosphorylation ${ }^{18}$. Filamentous actin should undergo depolymerization early in the acrosome reaction. In the present study, the density of $\beta$-actin was decreased in spermatozoa due to BPA exposure (Fig. 5A, B), suggesting that BPA downregulated $\beta$-actin in spermatozoa, and thus affected sperm motility, the acrosome reaction, and fertility. It has been reported that decreased actin abundance is associated with metastatic melanoma in mice ${ }^{45}$ and prostatic carcinoma in rats ${ }^{46}$.

GPX4 and PRDX5 are antioxidant enzymes that are predominantly expressed in spermatozoa as a response to stress. In somatic cells, GPX1, GPX4, PRDX3, and PRDX5 are responsible for consumption of approximately $99.9 \%$ of mitochondrial $\mathrm{H}_{2} \mathrm{O}_{2}{ }^{47}$. In mammalian spermatozoa, mitochondria are the principle site of ROS generation, which is strongly associated with male infertility ${ }^{48}$. Thus, failure of the antioxidant defense system in mitochondria to remove $\mathrm{H}_{2} \mathrm{O}_{2}$ leads ROS concentrations to reach toxic levels, and ultimately compromises normal sperm function and fertility. Therefore, the elevated levels of GPX4 and PRDX5 observed in the present study (Fig. 5A, B) were assumed to offer the sperm cells a survival advantages (ROS levels remained unchanged, see Supplemental Material, Fig. S2) in a BPA-containing microenvironment. Consistent with our findings, overexpression of PRDX5 has been reported in breast carcinoma ${ }^{49}$, hepatocellular carcinoma $^{50}$, and mesothelioma cells ${ }^{51}$.

GAPDH and SDHB are involved in glycolysis and the electron transport chain, respectively, and regulate sperm motility and male 

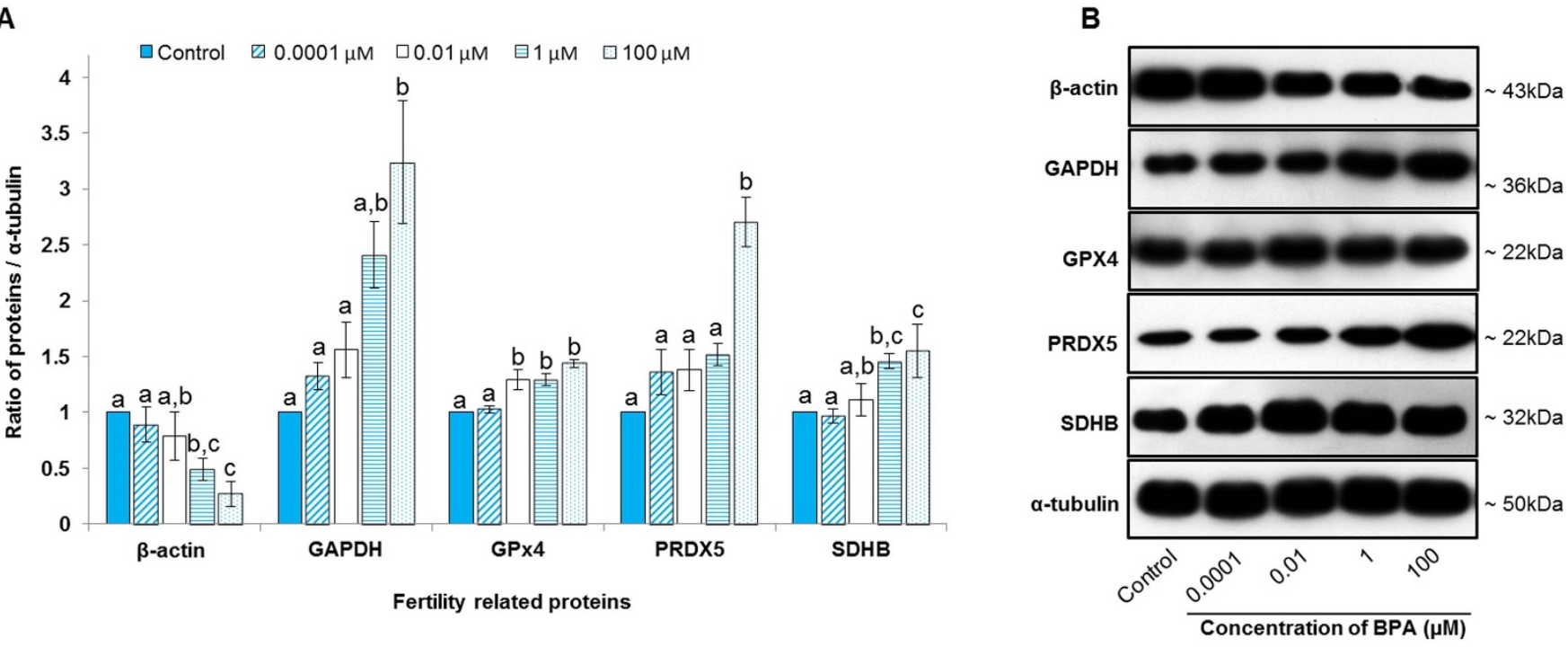

Figure 5 | Effect of bisphenol-A (BPA) on selected fertility-related proteins in spermatozoa. (A) Density of $\beta$-actin, glyceraldehyde-3-phosphate dehydrogenase (GAPDH), glutathione peroxidase-4 (GPX4), peroxiredoxin-5 (PRDX5) and succinate dehydrogenase (SDHB) in the control (blue bar) and BPA-treated (stripped bar: $0.0001 \mu \mathrm{M}$; open bar: $0.01 \mu \mathrm{M}$; straight stripped bar: $1 \mu \mathrm{M}$; and dotted bar: $100 \mu \mathrm{M}$ ) spermatozoa. (B) Selected fertilityrelated proteins were probed with specific antibodies and detected: $\beta$-actin, approximately $43 \mathrm{kDa}$; GAPDH, approximately $36 \mathrm{kDa}$; GPX4, approximately $22 \mathrm{kDa}$; PRDX5, approximately $22 \mathrm{kDa}$; and SDHB, approximately $32 \mathrm{kDa}$. Lane 1: control; lane 2: $0.0001 \mu \mathrm{M} \mathrm{BPA}$; lane 3: $0.01 \mu \mathrm{M} \mathrm{BPA}$; lane 4: $1 \mu \mathrm{M} \mathrm{BPA}$; and lane 5: $100 \mu \mathrm{M}$ BPA. Data are presented as mean \pm SEM $(n=3)$. Values with different superscript characters $\left({ }^{a, b, c}\right)$ indicate significant difference between the control and treatment groups as determined by one-way ANOVA $(P<0.05)$.

fertility ${ }^{21,22}$. Disruption of GAPDH expression by gene targeting can be used to block glycolysis in spermatozoa, which results in severe motility defects ${ }^{21}$. Xue et al. ${ }^{22}$ reported that the motility and viability of sperm were positively correlated with mitochondrial SDHB. Therefore, GAPDH and SDHB could influence mitochondrial function in spermatozoa and may serve as markers of sperm quality and male fertility. In the present study, BPA dose-dependently increased GAPDH and SDHB abundance; significant changes in protein abundance were produced by exposure to higher concentrations of BPA (Fig. 5A, B). These results suggest that high concentrations of BPA alter the function of GAPDH and SDHB, and could thus affect male fertility. Our results do not provide a clear explanation as to the manner in which ATP/motility are reduced by increased levels of GAPDHS/SDHB caused by exposure to $100 \mu \mathrm{M}$ BPA. However, the increased GAPDH/SDHB may have been insufficient to overcome BPA-mediated toxicity, and/or the substantial increase in expression resulted in atypical enzyme functioning. Enhanced glycolysis has been well established in cancer cells $s^{52}$. Because BPA may be carcinogenic $^{3}$, there might be a relationship between BPA-mediated increases in the levels of glycolytic enzymes and the occurrence of cancers in somatic cells. SDHB dysfunction is strongly associated with somatic cell tumorigenesis ${ }^{53}$. However, further studies are necessary to resolve this issue.

\section{Conclusions}

To our knowledge, this is the first comprehensive description of the in vitro effects of BPA on mouse spermatozoa. BPA at concentrations of $0.0001,0.01$, and $1 \mu \mathrm{M}$ did not produce significant or partial toxic effects on spermatozoa; however, $100 \mu \mathrm{M}$ BPA affected motility parameters, the acrosome reaction, fertilization, and early embryonic development, which are closely associated with down-regulation and phosphorylation of fertility-related proteins in spermatozoa. BPAinduced changes in the abundance of fertility-related proteins may be associated with increased health risk (Fig. 6).

\section{Methods}

Media and chemicals. All chemicals and reagents were purchased from SigmaAldrich (St. Louis, MO, USA), unless otherwise specified. Modified Tyrode's medium was used as a basic medium (BM), and prepared as previously described ${ }^{25,54}$. The culture medium was pre-incubated 1 day prior to the experiment and supplemented with bovine serum albumin (BSA; $4 \mathrm{mg} / \mathrm{mL}$ ) to induce capacitation. The BPA stock solution was diluted with dimethyl sulfoxide (DMSO) and stored in conical tubes at room temperature (RT). The stock solution was diluted with BM to achieve desired final concentrations of $0.0001,0.01,1$, and $100 \mu \mathrm{M}$. This concentration range was chosen because high doses of hormones (and estrogen mimics like BPA) can act through negative feedback loops to inhibit their own effects, and low doses are sufficient to exert their primary effects ${ }^{55}$. Additionally, exposure to environmentally relevant doses of BPA (approximately 1-3 nM) advances early and postnatal embryonic development ${ }^{56}$, whereas high concentrations $(100 \mu \mathrm{M})$ significantly decreased the development rate ${ }^{57}$.

Sperm collection, preparation, and exposure to BPA. All animal procedures were conducted according to the guidelines and were approved by the Institutional Animal Care and Use Committee of Chung-Ang University, Seoul, Korea. Experimental mice were kept in a room with controlled temperature $\left(22 \pm 2{ }^{\circ} \mathrm{C}\right)$, ventilation, and lighting (12-h light/dark cycles) and were provided ad libitum access to laboratory feed (Cargill Agripurina, Seongnam, Korea) and water. Spermatozoa were collected from sexually mature male ICR mice (Nara Biotech, Seoul, Korea) using a standard procedure ${ }^{25,31}$. Briefly, both cauda epididymides from each mouse were collected and the associated fat was removed. The sample was then placed on a piece of filter paper to remove any liquid. The cauda epididymides were placed in cell culture dishes with $\mathrm{BM}$, and punctured using a sterile needle to facilitate the release of spermatozoa. After preincubation, the sperm suspension was incubated in BM (containing $4 \mathrm{mg} / \mathrm{mL}$ BSA) supplemented with $0.0001,0.01,1$, and $100 \mu \mathrm{M}$ of BPA for $6 \mathrm{~h}$ in air at $37^{\circ} \mathrm{C}$. The control group consisted of DMSO-treated spermatozoa that did not contain BPA. For each of the independent experiments, three 3 male mice per replicate were considered.

Assessment of sperm motility and motion kinematic parameters. Sperm motility and kinematic parameters were measured using computer-assisted sperm analysis (CASA) (SAIS-PLUS version 10.1; Medical Supply, Seoul, Korea) as previously described $^{29}$. The $10 \times$ phase-contrast objective was used by the SAIS software to relay and analyze the spermatozoa, and 5 fields from each sample were randomly selected for evaluation of the movement of at least 250 sperm.

Measurement of cellular reactive oxygen species (ROS). Cellular ROS content was measured using the oxidation-sensitive fluorescent dye $2^{\prime}, 7^{\prime}$-dichlorofluorescin diacetate (DCFDA; Abcam, Cambridge, England) as previously described ${ }^{25}$. Briefly, after $6 \mathrm{~h}$ of incubation, the samples were washed and resuspended in $1 \mathrm{~mL}$ DCFDA mix and incubated at $37^{\circ} \mathrm{C}$ for $30 \mathrm{~min}$. Samples were resuspended in supplemental buffer after a second wash. Finally, the cell suspension $(500 \mu \mathrm{L})$ was seeded in a dark 96-well plate. Fluorescence was detected with a microplate fluorometer (Gemini Em; Molecular Devices, Sunnyvale, CA, USA) and quantified with SoftMax Pro 5 (Molecular Devices). The fluorescence of all treatment groups and the control group 

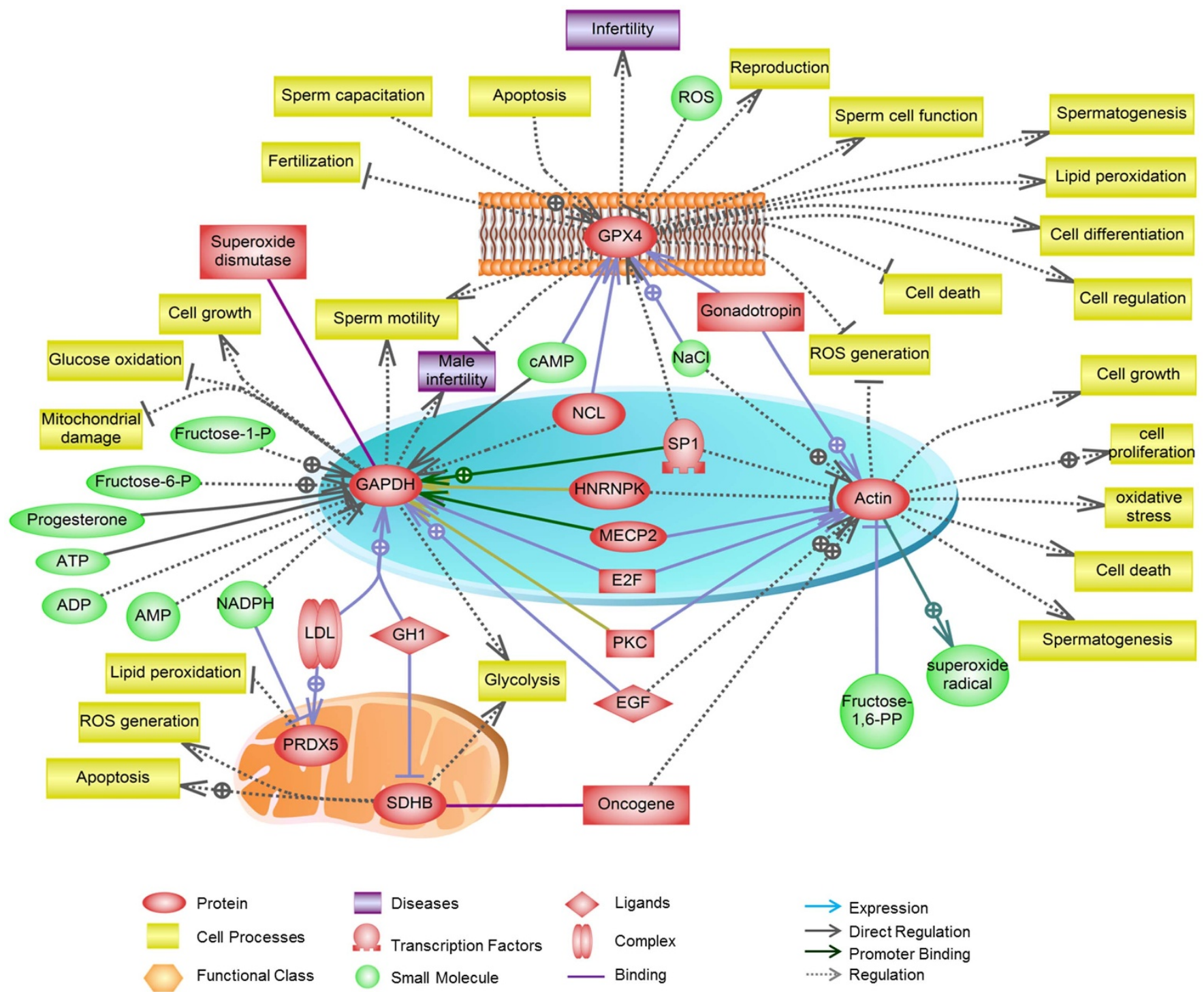

Figure 6 | Pathways regulated by selected fertility-related proteins in spermatozoa as predicted by Pathway Studio software. Regulated processes are represented by backgrounds of different colors (indicated in the figure). Regulatory events are displayed using arrows and documented by the figure, and were supported by the literature. The references linked to each protein were generated by the software and are not listed.

was calculated as a ratio $(485 \mathrm{~nm} / 535 \mathrm{~nm})$ and reported as the ratio of the fluorescence of the BPA-treated samples to that of the control samples.

Measurements of intracellular ATP generation. Cellular ATP was quantitatively detected using an ATP Bioluminescence Assay Kit (CLS II; Roche Molecular Biochemicals, Mannheim, Germany) according to the manufacturer's protocol and previously described methods ${ }^{25,31}$. Briefly, the treated cells were diluted $\left(10^{5}-\right.$ $10^{8}$ cells $/ \mu \mathrm{L}$ ), and $25 \mu \mathrm{L}$ of the diluted cells was added to each well of a 96 -well plate, to which $25 \mu \mathrm{L}$ of cell lysis reagent was added. The plate was incubated at RT for 5 min, diluted ATP $(50 \mu \mathrm{L})$ and luciferase reagent $(50 \mu \mathrm{L})$ were added, after which luminescence was detected using a Microplate Multimode Reader (GloMax ${ }^{\circledR}$-Multi Microplate Multimode Reader; Promega, Madison, WI, USA).

Assessment of sperm capacitation status. The capacitation status of the spermatozoa following BPA treatment was determined by a dual-staining method (combined Hoechst 33258/chlortetracycline fluorescence assessment) as described previously $y^{29,31}$. The spermatozoa were observed under a Microphot-FXA microscope (Nikon, Tokyo, Japan) with epifluorescence illumination using ultraviolet BP 340-380/LP 425 and BP 450-490/LP excitation/emission filters for H33258 and CTC, respectively. This dual-staining method analysis revealed 4 separate patterns among the spermatozoa, which were identified according to previously reported criteria ${ }^{58}$ : dead (D pattern, blue fluorescence distributed uniformly over the sperm head), live non-capacitated (F pattern, bright green fluorescence distributed uniformly over the sperm head), live capacitated (B pattern, bright green fluorescence over the acrosomal region and a dark postacrosomal region), and live acrosome-reacted (AR pattern, no fluorescence over the head, or green fluorescence only in the post-acrosomal region). At least 400 spermatozoa were evaluated per slide for each condition.

In vitro fertilization. Eight- to 12 -week-old B6D2F1/CrljOri hybrid female mice (Nara Biotech, Seoul, Korea) were super-ovulated by pregnant mare serum gonadotropin and human chorionic gonadotropin ${ }^{29,31}$. The oocytes were fertilized in vitro with control and BPA-treated spermatozoa according to previously described methods that were optimized for mice ${ }^{25,29}$. The fertilization rate was assessed by calculating the number of 2-cell embryos after $18 \mathrm{~h}$ of insemination. All embryos that developed to the blastocyst stage were counted.

Western blot analysis of phospho-PKA substrates, tyrosine phosphorylation, and a selected group of $\mathbf{5}$ fertility-related proteins. Mammalian spermatozoa must undergo capacitation and the acrosome reaction to achieve successful fertilization ${ }^{25,26}$, and both of these events are associated with the activation of cAMP/PKA-dependent signaling, which is followed by up-regulation of protein tyrosine phosphorylation ${ }^{25,27}$. Therefore, the PKA substrates and tyrosine phosphorylation of sperm proteins were assayed in current study according to previously described methods ${ }^{25,29}$. Briefly, a sample containing $500 \times 10^{6}$ cells $/ \mathrm{mL}$ from the treated spermatozoa was electrophoresed on a $10 \%$ SDS polyacrylamide gel and transferred to a polyvinylidene fluoride membrane (GE Healthcare, Pittsburgh, PA, USA). The membranes were blocked in 3\% ECL blocking agent (GE Healthcare). To detect PKA substrates, the membranes were incubated with an anti-phospho-PKA substrate rabbit polyclonal antibody ( $1: 10,000$; Cell Signaling Technology, Danvers MA, USA). The membranes were incubated with horseradish peroxidase (HRP)-conjugated goat anti-rabbit IgG (1:5,000; Abcam, Cambridge, England), and tyrosine phosphorylation was detected 
using an HRP-conjugated mouse monoclonal anti-phosphotyrosine antibody (PY20, $1: 2,500, \mathrm{Abcam}$ ). Western blots were also used to detect the fertility-related proteins, such as $\beta$-actin, GAPDH, GPX4, PRDX5, and SDHB to evaluate their role in spermatozoa after BPA exposure. Because, intensive studies on individual proteins are necessary to investigate the molecular basis of sperm function due to BPA exposure. For the detection of protein levels following BPA treatment, the membrane was blocked with $5 \%$ skim milk blocking agent. The proteins from treated spermatozoa were immunodetected after an overnight RT incubation with an anti- $\beta$ actin mouse monoclonal antibody (HRP-conjugated), an anti-GAPDH mouse monoclonal antibody, an anti-GPX4 rabbit polyclonal antibody, an anti-PRDX5 rabbit polyclonal antibody, or an anti-SDHB rabbit polyclonal antibody (all from Abcam), each of which was diluted with blocking solution $(1 \mu \mathrm{g} / \mathrm{mL})$. After primary incubation, the membranes were incubated in blocking solution with HRPconjugated goat anti-rabbit IgG (1:5,000; for GPX4, PRDX5, and SDHB; Abcam) or mouse IgG (for GAPDH) as a secondary antibody. $\alpha$-Tubulin expression was determined using a monoclonal anti $\alpha$-tubulin mouse antibody and used as an internal control for each protein. The proteins on the membranes were detected using enhanced chemiluminescence (ECL) reagents. All protein bands were scanned with a GS-800 calibrated imaging densitometer (Bio-Rad, Hercules, CA, USA) and analyzed with Quantity One software (Bio-Rad). Finally, the signal intensity ratios of the bands were calculated for phospho-PKA substrates, tyrosine phosphorylation species, $\beta$ actin, GAPDH, GPX4, PRDX5, and SDHB with respect to $\alpha$-tubulin.

Signaling pathway analysis. Pathway Studio (version 9.0, Ariadne Genomics, MD, USA) was used to predict the biological functions and signaling pathways associated with fertility-related proteins in spermatozoa ${ }^{14,26}$. The fertility-related proteins were entered into Pathway Studio in order to determine their associated signaling pathways, cellular functions, cellular regulation, and interactions with other proteins in spermatozoa.

Statistical analysis. Data were analyzed using one-way ANOVA in SPSS (version 12.0; IBM Corp., Armonk, NY, USA) and Tukey's test was used to determine the significance of differences between each group. Student's two-tailed t-test was employed to compare changes in motility (time-dependent) between the control and treatment groups. P-values $<0.05$ indicated statistical significance, and data are expressed as mean \pm SEM

1. Peretz, J. et al. Bisphenol A and reproductive health: update of experimental and human evidence, 2007-2013. Environ Health Perspect 122, 775-786 (2014).

2. Vandenberg, L. N., Hunt, P. A., Myers, J. P. \& Vom Saal, F. S. Human exposures to bisphenol A: mismatches between data and assumptions. Rev Environ Health 28, 37-58 (2013).

3. Soto, A. M. \& Sonnenschein, C. Environmental causes of cancer: endocrine disruptors as carcinogens. Nat Rev Endocrinol 6, 363-370 (2010).

4. Tiwari, D. \& Vanage, G. Mutagenic effect of Bisphenol A on adult rat male germ cells and their fertility. Reprod Toxicol 40, 60-68 (2013).

5. Doshi, T., D'souza, C. \& Vanage, G. Aberrant DNA methylation at Igf2-H19 imprinting control region in spermatozoaupon neonatal exposure to bisphenol A and its association with post implantation loss. Mol Biol Rep 40, 4747-4757 (2013).

6. Hulak, M., Gazo, I., Shaliutina, A. \& Linhartova, P. In vitro effects of bisphenol A on the quality parameters, oxidative stress, DNA integrity and adenosine triphosphate content in sterlet (Acipenser ruthenus) spermatozoa. Comp Biochem Physiol C Toxicol Pharmacol 158, 64-71 (2013).

7. Chitra, K. C., Latchoumycandane, C. \& Mathur, P. P. Induction of oxidative stress by bisphenol A in the epididymal sperm of rats. Toxicology 185, 119-127 (2003).

8. Lassen, T. H. et al. Urinary bisphenol A levels in young men: association with reproductive hormones and semen quality. Environ Health Perspect 122, 478-484 (2014).

9. Vilela, J. et al. Sperm impairments in adult vesper mice (Calomys laucha) caused by in utero exposure to bisphenol A. Andrologia, doi:10.1111/and.12182 (2013).

10. Mohamed, el-S. A. et al. The transgenerational impact of benzo(a)pyrene on murine male fertility. Hum Reprod 25, 2427-2433 (2010)

11. Mohamed, el-S. A. et al. Xenoestrogenic compounds promote capacitation and an acrosome reaction in porcine sperm. Theriogenology 75, 1161-1169 (2011).

12. Park, Y. J. et al. Xenoestrogenic chemicals effectively alter sperm functional behavior in mice. Reprod Toxicol 32, 418-424 (2011).

13. Yanagimachi, R. Mammalian fertilization. In: The Physiology of Reproduction (Knobil, E. \& Neill, J. D., eds). New York: Raven Press, 189-317 (1994).

14. Park, Y. J., Kim, J., You, Y. A. \& Pang, M. G. Proteomic revolution to improve tools for evaluating male fertility in animals. J Proteome Res 12, 4738-4747 (2013).

15. Park, Y. J., Kwon, W. S., Oh, S. A. \& Pang, M. G. Fertility-related proteomic profiling bull spermatozoa separated by percoll. J Proteome Res 11, 4162-4168 (2012)

16. Zhu, Y. F. et al. Proteomic analysis of effect of hyperthermia on spermatogenesis in adult male mice. J Proteome Res 5, 2217-2225 (2006).

17. Rahman, M. S., Lee, J. S., Kwon, W. S. \& Pang, M. G. Sperm proteomics: road to male fertility and contraception. Int J Endocrinol 2013, 360986, doi:10.1155/2013/ 360986 (2013).
18. Breitbart, H., Cohen, G. \& Rubinstein, S. Role of actin cytoskeleton in mammalian sperm capacitation and the acrosome reaction. Reproduction 129, 263-268 (2005).

19. O'Flaherty, C. The Enzymatic Antioxidant System of Human Spermatozoa. Advances in Andrology 2014, 626374, doi:10.1155/2014/626374 (2014).

20. Imai, H. et al. Failure of the expression of phospholipid hydroperoxide glutathione peroxidase in the spermatozoa of human infertile males. Biol Reprod 64, 674-683 (2001).

21. Miki, K. et al. Glyceraldehyde 3-phosphate dehydrogenase-S, a sperm-specific glycolytic enzyme, is required for sperm motility and male fertility. Proc Natl Acad Sci U S A 101, 16501-16506 (2004).

22. Xue, X. P., Shang, X. J., Fu, J., Chen, Y. G. \& Shi, Y. C. Detection and significance of succinate dehydrogenase of sperm mitochondria. Zhonghua Nan Ke Xue 9 , 601-603 (2003).

23. Donnelly, E. T., Lewis, S. E., McNally, J. A. \& Thompson, W. In vitro fertilization and pregnancy rates: the influence of sperm motility and morphology on IVF outcome. Fertil Steril 70, 305-314 (1998).

24. Oh, S. A., Park, Y. J., You, Y. A., Mohamed, E. A. \& Pang, M. G. Capacitation status of stored boar spermatozoa is related to litter size of sows. Anim Reprod Sci 121, 131-138 (2010).

25. Rahman, M. S. et al. Sodium nitroprusside suppresses male fertility in vitro. Andrology 2, 899-909 (2014).

26. Rahman, M. S., Kwon, W. S. \& Pang, M. G. Calcium influx and male fertility in the context of the sperm proteome: an update. Biomed Res Int 2014, 841615. doi:10.1155/2014/841615 (2014).

27. Visconti, P. E., Krapf, D., de la Vega-Beltrán, J. L., Acevedo, J. J. \& Darszon, A. Ion channels, phosphorylation and mammalian sperm capacitation. Asian J Androl 13, 395-405 (2011).

28. Thundathil, J. et al. Relationship between the proportion of capacitated spermatozoa present in frozen-thawed bull semen and fertility with artificial insemination. Int J Androl 6, 366-373 (1999).

29. Kwon, W. S. et al. Vasopressin effectively suppresses male fertility. PLoS One $\mathbf{8}$, e54192, doi:10.1371/journal.pone.0054192 (2013).

30. Luconi, M., Bonaccorsi, L., Forti, G. \& Baldi, E. Effects of estrogenic compounds on human spermatozoa: evidence for interaction with a nongenomic receptor for estrogen on human sperm membrane. Mol Cell Endocrinol 178, 39-45 (2001).

31. Shukla, K. K. et al. Nutlin-3a decreases male fertility via UQCRC2. PLoS One 8, e76959, doi:10.1371/journal.pone.0076959 (2013).

32. Chaveiro, A., Machado, L., Frijters, A., Engel, B. \& Woelders, H. Improvement of parameters of freezing medium and freezing protocol for bull sperm using two osmotic supports. Theriogenology 65, 1875-1890 (2006).

33. Wongtawan, T., Saravia, F., Wallgren, M., Caballero, I. \& Rodríguez-Martínez, H. Fertility after deep intra-uterine artificial insemination of concentrated lowvolume boar semen doses. Theriogenology 65, 773-787 (2006).

34. Oliveira, H., Spanò, M., Santos, C. \& Pereira, Mde. L. Lead chloride affects sperm motility and acrosome reaction in mice: lead affects mice sperm motility and acrosome reaction. Cell Biol Toxicol 25, 341-353 (2009).

35. Schumacher, J. et al. Evolutionary conservation of mammalian sperm proteins associates with overall, not tyrosine, phosphorylation in human spermatozoa. J Proteome Res 12, 5370-5382 (2013).

36. Secciani, F. et al. Protein profile of capacitated versus ejaculated human sperm. J Proteome Res 8, 3377-3389 (2009).

37. Wang, S. et al. Proteomic characteristics of human sperm cryopreservation. Proteomics 14, 298-310 (2014).

38. Oliva, R., de Mateo, S. \& Estanyol, J. M. Sperm cell proteomics. Proteomics 9 1004-1017 (2009).

39. Aitken, R. J. \& Baker, M. A. The role of proteomics in understanding sperm cell biology. Int J Androl 31, 295-302 (2008).

40. Zhao, C. et al. Role of translation by mitochondrial-type ribosomes during sperm capacitation: an analysis based on a proteomic approach. Proteomics 9, 1385-1399 (2009).

41. Gur, Y. \& Breitbart, H. Mammalian sperm translate nuclear-encoded proteins by mitochondrial-type ribosomes. Genes Dev 20, 411-416 (2006).

42. Yuan, J. Protein degradation and phosphorylation after freeze thawing result in spermatozoon dysfunction. Proteomics 14, 155-156 (2014).

43. McConnell, J. L. \& Wadzinski, B. E. Targeting protein serine/threonine phosphatases for drug development. Mol Pharmacol 75, 1249-1261 (2009).

44. Naz, R. K. \& Rajesh, P. B. Role of tyrosine phosphorylation in sperm capacitation/ acrosome reaction. Reprod Biol Endocrinol 2, 75, doi:10.1186/1477-7827-2-75 (2004).

45. Volk, T., Geiger, B. \& Raz, A. Motility and adhesive properties of high- and lowmetastatic murine neoplastic cells. Cancer Res 44, 11-24 (1984).

46. Zachary, J. M. et al. Actin filament organization of the Dunning R3327 rat prostatic adenocarcinoma system: correlation with metastatic potential. Cancer Res 46, 926-932 (1986).

47. Cox, B. A. G., Winterbourn, C. C. \& Hampton, M. B. Mitochondrial peroxiredoxin involvement in antioxidant defence and redox signaling. Biochem J 425, 313-325 (2010).

48. Aitken, R. J. \& De Iuliis, G. N. On the possible origins of DNA damage in human spermatozoa. Mol Hum Reprod 16, 3-13 (2009).

49. Noh, D. Y. et al. Overexpression of peroxiredoxin in human breast cancer. Anticancer Res 21, 2085-2090 (2001). 
50. Choi, J. H. et al. Overexpression of mitochondrial thioredoxin reductase and peroxiredoxin III in hepatocellular carcinomas. Anticancer Res 22, 3331-3335 (2002).

51. Kinnula, V. L. et al. Overexpression of peroxiredoxins I, II, III, V, and VI in malignant mesothelioma. J Pathol 196, 316-323 (2002).

52. Warburg, O. On the origin of cancer cells. Science 123, 309-314 (1956).

53. King, A., Selak, M. A. \& Gottlieb, E. Succinate dehydrogenase and fumarate hydratase: linking mitochondrial dysfunction and cancer. Oncogene 25, 4675-4682 (2006)

54. Quinn, P., Barros, C. \& Whittingham, D. G. Preservation of hamster oocytes to assay the fertilizing capacity of human spermatozoa. J Reprod Fertil 66, 161-168 (1982)

55. Hinterthuer, A. Safety dance over plastic. Sci Am 299, 110-111 (2008).

56. Takai, Y. et al. Preimplantation exposure to bisphenol A advances postnatal development. Reprod Toxicol 15, 71-74 (2001).

57. Takai. et al. Estrogen receptor-mediated effects of a xenoestrogen, bisphenol A, on preimplantation mouse embryos. Biochem Biophys Res Commun 270, 918-921 (2000).

58. Maxwell, W. M. \& Johnson, L. A. Physiology of spermatozoa at high dilution rates: the influence of seminal plasma. Theriogenology 52, 1353-1362 (1999)

\section{Acknowledgments}

This research was supported by a 2014 grant (14162MFDS661) from the Ministry of Food and Drug Safety of the Republic of Korea.

\section{Author contributions}

M.S.R., W.S.K., J.S.L. and S.J.Y. performed the experiments. M.S.R. and W.S.K. analyzed the data and performed the artwork. M.S.R. and M.G.P. draft the manuscript. Both M.G.P. and B.Y.R. supervised the design of study and revised the manuscript. All authors critically reviewed the manuscript for intellectual content and gave final approval for the version to be published.

\section{Additional information}

Supplementary information accompanies this paper at http://www.nature.com/ scientificreports

Competing financial interests: The authors declare no competing financial interests.

How to cite this article: Rahman, M.S. et al. Bisphenol-A Affects Male Fertility via Fertility-related Proteins in Spermatozoa. Sci. Rep. 5, 9169; DOI:10.1038/srep09169 (2015).

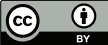

This work is licensed under a Creative Commons Attribution 4.0 International License. The images or other third party material in this article are included in the article's Creative Commons license, unless indicated otherwise in the credit line; if the material is not included under the Creative Commons license, users will need to obtain permission from the license holder in order to reproduce the material. To view a copy of this license, visit http://creativecommons.org/licenses/by/4.0/ 